\title{
A Novel Approach to Studying Heterogeneous Mineral Calcite Nucleation and Growth
}

\author{
KABIR RAHEEM $^{1}$, OLUJIDE SANNI ${ }^{2}$, THIBAUT
}

CHARPENTIER $^{3}$, ANNE NEVILLE ${ }^{4}$

${ }^{1}$ School of Mechanical Engineering, University of Leeds, United Kingdom. K.Raheem@leeds.ac.uk

${ }^{2}$ School of Mechanical Engineering, University of Leeds, United Kingdom. O.S.Sanni@leeds.ac.uk

${ }^{3}$ School of Chemical and Process Engineering, university of Leeds, United Kingdom. T.Charpentier@leeds.ac.uk

${ }^{4}$ School of Mechanical Engineering, University of Leeds, United Kingdom. A.Neville@leeds.ac.uk

The precipitation of calcite in reservoirs and importantly on surface facilities is one of the most significant challenges encountered in oil and gas exploration and production. Recent studies have shown that mineral calcite nucleation and growth on a surface is not necessarily a result of bulk solution crystallization and that they often do not share the same trends with regards to crystal growth kinetics and inhibition. It is important to develop fundamental understanding of mechanisms of mineral calcite formation on components' surfaces.

In this study, a newly developed novel capillary flow rig, was used to evaluate heterogeneous surface precipitation of $\mathrm{CaCO}_{3}$ (calcite) under dynamic flowing conditions. An important characteristic of this set-up is that saturation ratio (SR) regarded as the "thermodynamic driving force" for crystal nucleation remains constant and that there are no bulk crystals in the solution. This allows the decoupling of bulk and surface scaling, providing a better mechanic understanding of how scale cryatsl precipitate directly and build up on a surface.

Two surfaces were tested, as-received and functionalized stainless steel as a result of their wettability; and by extension surface energy. Stainless steel samples were functionalized by the application of silane ((3-Aminopropy)triethoxysilane) via vacuum deposition. The results shed new insight into characteristics of a surface (conditions and properties) that influence heterogeneous mineral crystal formation and what makes a good anti-fouling / anti-scaling substrate. 